\title{
Nonlinear Measurement Update and Prediction: Prior Density Splitting Mixture Estimator
}

\author{
Andreas Rauh, Kai Briechle, and Uwe D. Hanebeck Member, IEEE
}

\begin{abstract}
In this paper, the Prior Density Splitting Mixture Estimator (PDSME), a new Gaussian mixture filtering algorithm for nonlinear dynamical systems and nonlinear measurement equations, is introduced. This filter reduces the linearization error which typically arises if nonlinear state and measurement equations are linearized to apply linear filtering techniques. For that purpose, the PDSME splits the prior probability density into several components of a Gaussian mixture with smaller covariances. The PDSME is applicable to both prediction and filter steps. A measure for the linearization error similar to the Kullback-Leibler distance is introduced allowing the user to specify the desired estimation quality. An upper bound for the computational effort can be given by limiting the maximum number of Gaussian mixture components.
\end{abstract}

\section{INTRODUCTION}

Common techniques for state and parameter estimation for nonlinear systems with stochastic uncertainties rely on the discretization of the state space using fixed or variable grids and particle filters, see e.g. [1]-[4]. These techniques are only applicable to low-dimensional systems since the computational effort increases exponentially with the dimension of the state space. Major problems are the necessity for adaptive grid sizes as well as variable numbers of grid points and particles to ensure successful recursive filtering. Since the estimated probability density function (PDF) is represented by small numbers of particles in domains with small probability, resampling techniques are unavoidable to guarantee sufficient estimation quality for all possible states.

Other methods for recursive, nonlinear filtering rely on the approximation of PDFs by parameterizable functions. Typically, Gaussian PDFs defined by mean and covariance are assumed. Furthermore, algorithms using exponential PDFs have been investigated in related work [5]. For nonlinear systems, the assumption of a Gaussian PDF is always suboptimal if multi-modal or asymmetric PDFs which are significantly different from a Gaussian have to be represented.

One possibility to calculate the mean and covariance of Gaussian approximations of the posterior PDF is the numerical integration of the exact non-Gaussian posterior PDF which is defined by the formula of Bayes [6], [7]. Usually, this involves integration of non-Gaussian PDFs over infinite

Andreas Rauh is with the Chair of Mechatronics, University of Rostock, D-18059 Rostock, Germany, Andreas.Rauh@uni-rostock.de. Kai Briechle is with Dr. Johannes Heidenhain GmbH, D-83301 Traunreut, Germany, Briechle@heidenhain.de. Uwe D. Hanebeck is with the Institute of Computer Science and Engineering, Universität Karlsruhe (TH), D-76128 Karlsruhe, Germany, Uwe. Hanebeck@ieee. org.

Parts of this work were performed while all authors were with the Institute of Automatic Control Engineering, Technische Universität München, Munich, Germany. intervals of a multi-dimensional state space. To avoid numerical integration, Gaussian filters often rely on one of the following approximations. (i) It is assumed that the prior PDFs of the state variables, their nonlinear transformations by the measurement or state equations, and the noise densities are jointly Gaussian distributed [8]. (ii) Nonlinear system models are replaced by linearized measurement and state equations to apply the so-called Extended Kalman Filter (EKF) and to approximate the posterior density. The well-known Kalman filter [9] corresponds to the exact solution of Bayesian state estimation for linear systems with white Gaussian noise.

In this paper, a new approach of improving the estimation quality of Gaussian mixture filtering algorithms [10], which are based on the linearization of the measurement and state equations, is introduced. For each component of the prior PDF (represented by a Gaussian mixture density), the corresponding posterior is approximated after linearization of both the measurement and state equations. If a measure quantifying the approximation quality exceeds a user-defined bound, the number of prior density components is increased by a novel splitting procedure. During splitting selected terms of the Gaussian mixture are subdivided into components with smaller covariances. For other algorithms based on splitting or merging of PDFs, see e.g. [11], [12].

In Section II, a precise problem formulation is given. In Section III, an overview of the proposed filtering algorithm is presented. Some measures for the distance between PDFs are reviewed in Section IV to determine the estimation quality of the proposed algorithm. In Section V, the new Prior Density Splitting Mixture Estimator (PDSME) is presented. The filter step for nonlinear models of measurement processes is derived in the Subsections V-A to V-D. In Subsection V-E, the prediction step of the PDSME is described for nonlinear state equations. In Section VI, selected examples are presented to demonstrate the performance of the PDSME. Finally, in Section VII, the paper is concluded.

\section{Problem Formulation}

In this paper, Bayesian state estimation for multidimensional nonlinear discrete-time systems is considered. These systems consist of (nonlinear) state equations

$$
\underline{x}_{k+1}=\underline{a}_{k}\left(\underline{x}_{k}\right)+\underline{w}_{k}
$$

and (nonlinear) models of measurement processes

$$
\underline{\hat{y}}_{k}=\underline{h}_{k}\left(\underline{x}_{k}\right)+\underline{v}_{k}
$$

with $\underline{a}_{k}: D \mapsto \mathbb{R}^{n}, \underline{a}_{k} \in C^{1}\left(D, \mathbb{R}^{n}\right), \underline{h}_{k}: D \mapsto \mathbb{R}^{z}, \underline{h}_{k} \in$ $C^{1}\left(D, \mathbb{R}^{z}\right)$, and $D \subset \mathbb{R}^{n}$ open. 
Throughout this paper, the system noise $\underline{w}_{k}$ and the measurement noise $\underline{v}_{k}$ are normally distributed with $f_{w, k}\left(\underline{w}_{k}\right)=$ $\mathscr{N}\left(\underline{\mu}_{w, k}, \mathbf{C}_{w, k}\right)$ and $f_{v, k}\left(\underline{v}_{k}\right)=\mathscr{N}\left(\underline{\mu}_{v, k}, \mathbf{C}_{v, k}\right)$, resp., where $\mathscr{N}(\underline{\mu}, \mathbf{C})$ denotes a Gaussian PDF with mean $\underline{\mu}$ and covariance $\mathbf{C}$.

The estimation process can be subdivided into filter and prediction steps. In the filter step, the state estimate is updated according to the formula of Bayes

$$
f_{x, k}^{e}\left(\underline{x}_{k} \mid \underline{\hat{y}}_{k}\right)=\frac{f_{x, k}^{p}\left(\underline{x}_{k}\right) f_{v, k}\left(\underline{\hat{y}}_{k}-\underline{h}_{k}\left(\underline{x}_{k}\right)\right)}{\int_{\mathbb{R}^{n}} f_{x, k}^{p}\left(\underline{x}_{k}\right) f_{v, k}\left(\underline{\hat{y}}_{k}-\underline{h}_{k}\left(\underline{x}_{k}\right)\right) d \underline{x}_{k}}
$$

by considering the measured data $\hat{y}_{k}$ [6], [7]. Prior knowledge about uncertainties of the state vector $\underline{x}_{k}$ is described by the density $f_{x, k}^{p}\left(\underline{x}_{k}\right)$. Equation (3) provides the exact solution for the posterior PDF $f_{x, k}^{e}\left(\underline{x}_{k} \mid \underline{y}_{k}\right)$.

According to formula (3), the exact posterior PDF $f_{x, k}^{e}\left(\underline{x}_{k} \mid \underline{\hat{y}}_{k}\right)$ does not remain Gaussian for nonlinear measurement equations $\underline{h}_{k}\left(\underline{x}_{k}\right)$, even if both $f_{x, k}^{p}$ and $f_{v, k}$ are Gaussian. The analytic expression for the posterior PDF is becoming more and more complicated with each additional measured value used for the recursive update of the state estimate.

Furthermore, the theoretically exact solution of the prediction step, handling the dynamics of nonlinear state equations (1), involves the evaluation of multi-dimensional convolution integrals

$$
f_{x, k+1}^{p}\left(\underline{x}_{k+1}\right)=\int_{\mathbb{R}^{n}} f_{x, k}^{e}\left(\underline{x}_{k}\right) f_{w, k}\left(\underline{x}_{k+1}-\underline{a}_{k}\left(\underline{x}_{k}\right)\right) d \underline{x}_{k} .
$$

In general, the predicted PDF $f_{x, k+1}^{p}\left(\underline{x}_{k+1}\right)$ can only be computed numerically. Analytic solutions only exist for a few special cases such as Gaussian or Gaussian mixture densities $f_{x, k}^{e}\left(\underline{x}_{k}\right)$ with linear functions $\underline{a}_{k}\left(\underline{x}_{k}\right)$.

To avoid numerical evaluation of multi-dimensional convolution integrals, efficient methods for the approximation of the PDFs (3) and (4) are derived in the following. Although, the case of Gaussian system and measurement noise is considered, the generalization to non-Gaussian noise described by Gaussian mixture representations is straightforward yielding a weighted superposition of the results obtained for Gaussian noise.

To describe the posterior PDF $f_{x, k}^{e}\left(\underline{x}_{k} \mid \underline{\hat{y}}_{k}\right)$, approximations using Gaussian mixture densities

$$
\begin{aligned}
f_{x, k}^{e}\left(\underline{x}_{k} \mid \underline{\hat{y}}_{k}\right) & =\sum_{j=1}^{L} f_{x, k}^{e, j}\left(\underline{x}_{k}\right) \\
& =\sum_{j=1}^{L} \omega_{x, k}^{e, j} \frac{\exp \left[-\frac{1}{2}\left\|\underline{x}_{k}-\underline{\mu}_{x, k}^{e, j}\right\|_{\left.\left(\mathbf{C}_{x x, k}^{e, j}\right)^{-1}\right]}^{2}\right.}{\sqrt{(2 \pi)^{n}\left|\mathbf{C}_{x x, k}^{e, j}\right|}}
\end{aligned}
$$

with a variable number of $L$ components are determined. Analogously, the predicted PDF $f_{x, k+1}^{p}\left(\underline{x}_{k+1}\right)$ is approximated by a Gaussian mixture defined by the weighting factors $\omega_{x, k+1}^{p, j}$, the means $\underline{\mu}_{x, k+1}^{p, j}$, and the covariances $\mathbf{C}_{x, k+1}^{p, j}$.

\section{The Proposed Filtering Algorithm: PDSME}

In this Section, an overview of the proposed PDSME is given. For both the filter and prediction step three components of the algorithm are defined. These components are a splitting procedure for the prior density, the calculation of a filter step for Gaussian mixtures using a bank of EKFs, and the merging of posterior Gaussian mixture components. To simplify the introduction of these components, only the filter step (upper part of Fig. 1) is described in this Section. The prediction step (lower part of Fig. 1) consists of the same three components.

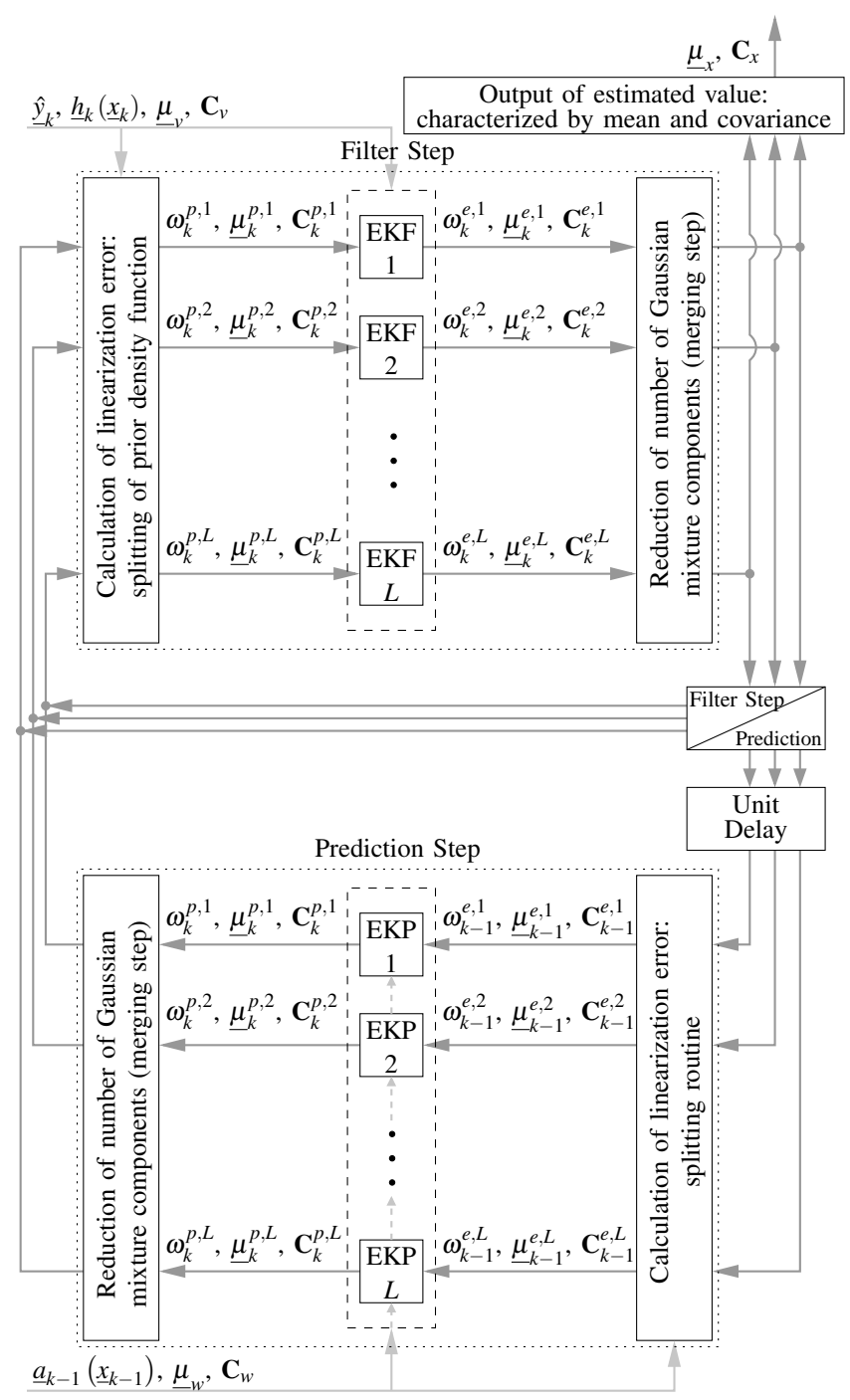

Fig. 1. Overview of the PDSME algorithm: linearized filter step (upper part) and linearized prediction step (lower part).

First, for each component of the prior PDF described by a Gaussian mixture with $L \geq 1$ components, a measure quantifying the linearization error is computed. This measure compares the approximated posterior PDF to the exact result of the estimation step. The approximation is calculated after replacement of the nonlinear measurement equation $\underline{h}_{k}\left(\underline{x}_{k}\right)$ by its linearization $\underline{h}_{k}\left(\underline{x}_{k}\right)$, evaluated at the means $\underline{\mu}_{x, k}^{p, j}, j=$ $1, \ldots, L$, of the prior PDF. The measure for the linearization 
error, which is similar to the Kullback-Leibler distance [13], is minimized by splitting components of the prior PDF to achieve a user-defined estimation quality.

Second, the filter step is evaluated for each Gaussian mixture component using a bank of EKFs. This leads to a Gaussian mixture representation of the density after the measurement update. For nonlinear measurement models, the number of mixture components is usually increased compared to the prior PDF.

Hence, a merging step is introduced as the third component of the PDSME to reduce redundancy in the density representation as well as the number of mixture components. The merging step is the prerequisite to use the PDSME recursively. It prevents an unlimited growth of the number of Gaussian mixture components. An upper bound for the computational effort can be specified by limiting the maximum number of components approximating the posterior PDFs.

For the purpose of recursive state estimation, this filter step is followed by a sequence of further filter and prediction steps, resp. As shown in the lower part of Fig. 1, the prediction step consists of the same components as the filter step. In Section V, further details on the calculation of both the filter and prediction step of the PDSME are given.

\section{Distance Between Probability Densities}

Calculating a measure for the distance between the exact PDF $f(\underline{x})$ and its Gaussian approximation $\bar{f}(\underline{x})$ is one of the main aspects of the PDSME. This section gives a brief summary of some measures similar to the Kullback-Leibler distance [13] that can be used for this purpose.

The Kullback-Leibler distance between two PDFs $f(\underline{x})$ and $\bar{f}(\underline{x})$ is defined by

$$
\mathscr{D}_{K L}(\bar{f} \| f):=\int_{\mathbb{R}^{n}} \bar{f}(\underline{x}) \ln \left(\frac{\bar{f}(\underline{x})}{f(\underline{x})}\right) d \underline{x}
$$

or, alternatively, by $\mathscr{D}_{K L}(f \| \bar{f})$. Obviously, the KullbackLeibler distance is not symmetric, i.e., $\mathscr{D}_{K L}(\bar{f} \| f) \neq$ $\mathscr{D}_{K L}(f \| \bar{f})$ because this distance measure depends upon the support of the two PDFs that are compared. Symmetry can e.g. be achieved by the arithmetic average

$$
\mathscr{J}(\bar{f}, f):=\frac{1}{2}\left(\mathscr{D}_{K L}(\bar{f} \| f)+\mathscr{D}_{K L}(f \| \bar{f})\right)
$$

of $\mathscr{D}_{K L}(\bar{f} \| f)$ and $\mathscr{D}_{K L}(f \| \bar{f})$, see Jeffreys' distance in [14], or by the resistor-average distance [15]

$$
\mathscr{R}(\bar{f}, f):=\left(\frac{1}{\mathscr{D}_{K L}(\bar{f} \| f)}+\frac{1}{\mathscr{D}_{K L}(f \| \bar{f})}\right)^{-1}
$$

For identically normalized PDFs $f(\underline{x})$ and $\bar{f}(\underline{x})$, the Kullback-Leibler distance is always non-negative, i.e., $\mathscr{D}_{K L}(\bar{f} \| f) \geq 0$ and $\mathscr{D}_{K L}(f \| \bar{f}) \geq 0$ hold. Then, $\mathscr{D}_{K L}(\bar{f} \| f)=$ $\mathscr{D}_{K L}(f \| \bar{f})=0$ also only holds for $f(\underline{x}) \equiv \bar{f}(\underline{x})$. In this paper, identical normalization of the exact and approximated posterior mixture components is not guaranteed. Thus, alternative, non-negative definitions (corresponding to cost functions to be minimized) are required which provide information about the components of the prior PDF which are mapped worst onto the posterior.

The definition (6), and therefore also (7) and (8), involve the integration of the exact, non-Gaussian posterior PDF $f(\underline{x})$ defined by the formula of Bayes. Since these integrals can only be evaluated analytically for a few special cases and since symmetry is of minor importance in this application, the definition

$$
\mathscr{D}_{2 i}(\bar{f} \| f):=\int_{\mathbb{R}^{n}} \bar{f}(\underline{x})\left(\ln \left(\frac{\bar{f}(\underline{x})}{f(\underline{x})}\right)\right)^{2 i} d \underline{x}
$$

with $i \in \mathbb{N}$, obtained by modification of (6), is introduced. It allows to detect the mixture component with the largest deviation between $f(\underline{x})$ and $\bar{f}(\underline{x})$. In the following, only the case $i=1$ is considered. As shown in Subsection VI-A, the value of this integral can be calculated analytically for polynomial and trigonometric measurement equations (involving sine and cosine) as a linear combination of the moments of the Gaussian density $\bar{f}(x)$.

\section{Prior Density Splitting Mixture Estimator}

In the Subsections V-A to V-D, the filter step of the PDSME is described. Then, in Subsection V-E, the prediction step of the PDSME is presented.

\section{A. Linearization Error in the Filter Step}

At the beginning of the filter step, the linearization error $\mathscr{D}_{2}\left(\bar{f}_{x, k}^{e, j} \| f_{x, k}^{e, j}\right)$ according to (9) is calculated for each component $f_{x, k}^{p, j}\left(\underline{x}_{k}\right), j=1, \ldots, L$, of the prior density $f_{x, k}^{p}\left(\underline{x}_{k}\right)$. The Gaussian mixture component

$$
j_{\text {max }}:=\underset{j=1, \ldots, L}{\arg \max }\left\{\mathscr{D}_{2}\left(\bar{f}_{x, k}^{e, j} \| f_{x, k}^{e, j}\right)\right\}
$$

is identified and split into $L_{R}$ components with smaller covariances to reduce the linearization error. Splitting is applied as long as the total linearization error

$$
\mathscr{D}_{2, \text { sum }}:=\sum_{j=1}^{\tilde{L}} \mathscr{D}_{2}\left(\bar{f}_{x, k}^{e, j} \| f_{x, k}^{e, j}\right) \geq \varepsilon_{1}
$$

and the maximum linearization error $\mathscr{D}_{2, \max } \geq \varepsilon_{2}$ of the Gaussian mixture with the increased number of $\tilde{L}=L+$ $L_{R}-1$ components exceed bounds $\varepsilon_{1}$ and $\varepsilon_{2}$ representing the approximation quality specified by the user.

\section{B. Splitting of the Prior Density Function}

To reduce the on-line computational effort, the splitting library required for replacement of $f_{x, k}^{p, j_{\max }}\left(\underline{x}_{k}\right)$ by $L_{R}$ components with smaller covariances, has been optimized off-line, such that a standard normal distribution of a scalar variable can be replaced by a Gaussian mixture approximation with $L_{R}$ components. This splitting library is defined by the weighting factors $\omega_{R, \zeta}$, means $\mu_{R, \zeta}$, and standard deviations $\sigma_{R, \zeta}$ for all $\zeta=1, \ldots, L_{R}$.

Example V.1 For $L_{R}=4$, a set of parameters of a splitting library is given in Tab. I. In Section VI, these parameters are used to demonstrate the performance of the PDSME. 
TABLE I

SPLITTING LIBRARY WITH $L_{R}=4$ MIXTURE COMPONENTS.

\begin{tabular}{|c||r|r|c|c|}
\hline$\zeta$ & \multicolumn{1}{|c|}{1} & \multicolumn{1}{|c|}{2} & 3 & 4 \\
\hline$\omega_{R, \zeta}$ & 0.093 & 0.407 & 0.407 & 0.093 \\
\hline$\mu_{R, \zeta}$ & -1.407 & -0.447 & 0.447 & 1.407 \\
\hline$\sigma_{R, \zeta}$ & 0.675 & 0.675 & 0.675 & 0.675 \\
\hline
\end{tabular}
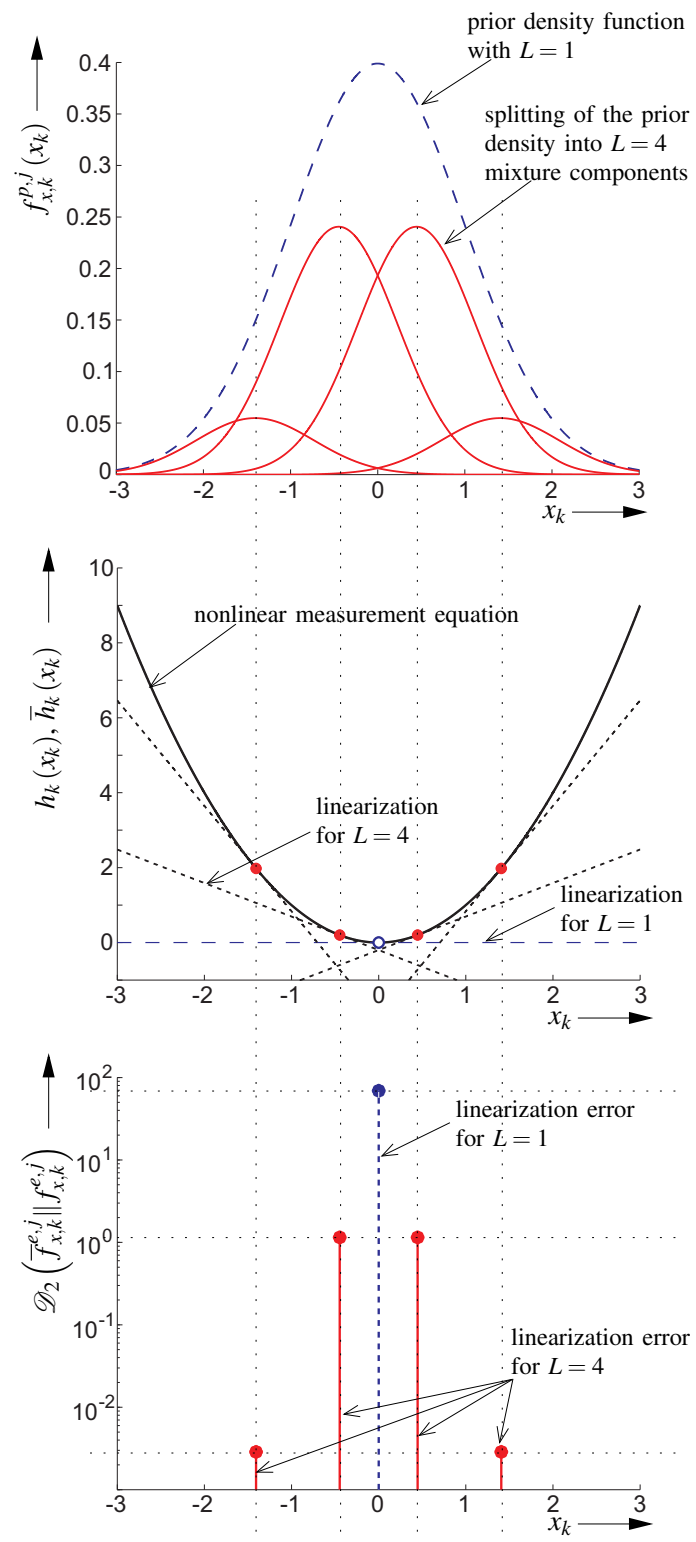

Fig. 2. Reduction of the linearization error by prior density splitting.

Example V.2 In Fig. 2, the key idea of the PDSME to reduce the linearization error by splitting the prior PDF into a Gaussian mixture with smaller covariances is shown. First, a Gaussian PDF with $\mu_{x, k}^{p}=0.0$ and $\sigma_{x, k}^{p}=1.0$ is replaced by $L_{R}=4$ Gaussian mixture components with the parameters from Tab. I. Second, the linearization of the measurement equation $\hat{y}_{k}=x_{k}^{2}+v_{k}$ at the means $\mu_{x, k}^{p, j}, j=1, \ldots, L$, is depicted for both representations of the PDF, i.e., for $L=1$ and $L=4$. Third, the corresponding linearization errors are shown for each component of the PDF with $\mu_{v}=0.0$, $\sigma_{v}=0.5$, and $\hat{y}_{k}=0.75$. In this example, the maximum linearization error $\mathscr{D}_{2, \max }$ is reduced from 69.162 to 1.150 . Furthermore, the total error $\mathscr{D}_{2, \text { sum }}$ is reduced from 69.162 to 2.305 by splitting of the prior PDF into 4 components.

For a non-axis-parallel Gaussian mixture component, characterized by $\omega_{x, k}^{p, j_{\max }}, \underline{\mu}_{x, k}^{p, j_{\max }}$, and $\mathbf{C}_{x x, k}^{p, j_{\max }}$, splitting is performed according to

$$
\begin{gathered}
\omega_{x, k}^{p, \alpha_{1}, \ldots, \alpha_{n}}=\omega_{x, k}^{p, j_{\max }} \cdot \prod_{i=1}^{n} \omega_{R, \alpha_{i}}, \\
\underline{\mu}_{x, k}^{p, \alpha_{1}, \ldots, \alpha_{n}}=\mathbf{P}_{x x, k}^{p, j_{\max }} \cdot\left[\begin{array}{c}
\mu_{R, \alpha_{1}} \\
\vdots \\
\mu_{R, \alpha_{n}}
\end{array}\right]+\underline{\mu}_{x, k}^{p, j_{\max }}, \text { and } \\
\mathbf{C}_{x x, k}^{p, \alpha_{1}, \ldots, \alpha_{n}}=\mathbf{P}_{x x, k}^{p, j_{\max }} \cdot \operatorname{diag}\left(\left[\begin{array}{c}
\sigma_{R, \alpha_{1}}^{2} \\
\vdots \\
\sigma_{R, \alpha_{n}}^{2}
\end{array}\right]\right) \cdot\left(\mathbf{P}_{x x, k}^{p, j_{\max }}\right)^{T},
\end{gathered}
$$

where $\mathbf{P}_{x x, k}^{p, j_{\max }}$ denotes the Cholesky decomposition of $\mathbf{C}_{x x, k}^{p, j_{\max }}$. The original component $f_{x, k}^{p, j_{\max }}\left(\underline{x}_{k}\right)$ of the prior density is replaced by $\left(L_{R}\right)^{n}$ components denoted by the indices $\alpha_{i}=$ $1, \ldots, L_{R}$ with $i=1, \ldots, n$. The increase in the number of mixture components can be limited if splitting is restricted to selected components of the state vector.

\section{Calculation of the Filter Step}

In the filter step (upper part of Fig. 1), the measurement equation $\underline{h}_{k}\left(\underline{x}_{k}\right)$ is replaced by its linearization

$$
\underline{h}_{k}\left(\underline{x}_{k}\right) \approx \underline{\bar{h}}_{k}\left(\underline{x}_{k}\right):=\underline{H}_{0}^{j}\left(\underline{\mu}_{x, k}^{p, j}\right)+\mathbf{H}_{1}^{j}\left(\underline{\mu}_{x, k}^{p, j}\right) \cdot \underline{x}_{k}
$$

at the means $\underline{\mu}_{x, k}^{p, j}, j=1, \ldots, L$, of all components of the prior density $f_{x, k}^{p}\left(\underline{x}_{k}\right)$ with

$$
\mathbf{H}_{1}^{j}:=\mathbf{H}_{1}^{j}\left(\underline{\mu}_{x, k}^{p, j}\right)=\left.\frac{\partial \underline{h}_{k}\left(\underline{x}_{k}\right)}{\partial \underline{x}_{k}}\right|_{\underline{x}_{k}=\underline{\mu}_{x, k}^{p, j}}
$$

and

$$
\underline{H}_{0}^{j}:=\underline{H}_{0}^{j}\left(\underline{\mu}_{x, k}^{p, j}\right)=\underline{h}_{k}\left(\underline{\mu}_{x, k}^{p, j}\right)-\mathbf{H}_{1}^{j}\left(\underline{\mu}_{x, k}^{p, j}\right) \cdot \underline{\mu}_{x, k}^{p, j} .
$$

Using a bank of EKFs, the approximation $\mathscr{N}\left(\underline{\mu}_{x, k}^{e, j}, \mathbf{C}_{x x, k}^{e, j}\right)$ of each component of the posterior PDF is given by the mean

$$
\underline{\mu}_{x, k}^{e, j}=\underline{\mu}_{x, k}^{p, j}+\mathbf{K}_{k} \cdot\left(\underline{\hat{y}}_{k}-\underline{H}_{0}^{j}-\mathbf{H}_{1}^{j} \underline{\mu}_{x, k}^{p, j}-\underline{\mu}_{v}\right)
$$

and the covariance

$$
\mathbf{C}_{x x, k}^{e, j}=\mathbf{C}_{x x, k}^{p, j}-\mathbf{K}_{k} \mathbf{H}_{1}^{j} \mathbf{C}_{x x, k}^{p, j}
$$

with

$$
\mathbf{K}_{k}:=\mathbf{C}_{x x, k}^{p, j}\left(\mathbf{H}_{1}^{j}\right)^{T}\left[\mathbf{H}_{1}^{j} \mathbf{C}_{x x, k}^{p, j}\left(\mathbf{H}_{1}^{j}\right)^{T}+\mathbf{C}_{v}\right]^{-1} .
$$

The result of the filter step is therefore a Gaussian mixture

$$
\bar{f}_{x, k}^{e}\left(\underline{x}_{k}\right)=\sum_{j=1}^{L} \omega_{x, k}^{e, j} \mathscr{N}\left(\underline{\mu}_{x, k}^{e, j}, \mathbf{C}_{x x, k}^{e, j}\right)
$$


with the weighting factors

$$
\begin{aligned}
\omega_{x, k}^{e, j}= & \omega_{x, k}^{p, j} \sqrt{\frac{\left|\mathbf{C}_{x x, k}^{e, j}\right|}{\left|\mathbf{C}_{x x, k}^{p, j}\right|} \exp \left[-\frac{1}{2}\left\|\underline{\mu}_{x, k}^{e, j}-\underline{\mu}_{x, k}^{p, j}\right\|_{\left(\mathbf{C}_{x x, k}^{e, j}\right)^{-1}}^{2}\right]} \\
& \frac{\exp \left[-\frac{1}{2}\left\|\underline{\hat{y}}_{k}-\underline{H}_{0}^{j}-\mathbf{H}_{1}^{j} \underline{\mu}_{x, k}^{e, j}-\underline{\mu}_{v}\right\|_{\left(\mathbf{C}_{v}\right)^{-1}}^{2}\right]}{\sqrt{(2 \pi)^{z}\left|\mathbf{C}_{v}\right|}} .
\end{aligned}
$$

\section{Merging of Posterior Mixture Components}

In the merging step, groups of two or more overlapping Gaussian mixture components of the posterior PDF are replaced by a single Gaussian component, if the additional error introduced by this merging step is negligible.

For that purpose, all possible candidates for replacement are determined: After interpreting the components $\bar{f}_{x, k}^{e, j}\left(\underline{x}_{k}\right)$, $j=1, \ldots, L$, of the Gaussian mixture $\bar{f}_{x, k}^{e}\left(\underline{x}_{k}\right)$ as the vertices of a complete graph $\mathscr{G}$, the Mahalanobis distance [16]

$$
\mathscr{M}_{i, j}=\left\|\underline{\mu}_{x, k}^{e, i}-\underline{\mu}_{x, k}^{e, j}\right\|_{\left(\mathbf{C}_{x x, k}^{e, i}+\mathbf{C}_{x x, k}^{e, j}\right)^{-1}}^{2}
$$

between two different Gaussian mixture components $\bar{f}_{x, k}^{e, i}\left(\underline{x}_{k}\right)$ and $\bar{f}_{x, k}^{e, j}\left(\underline{x}_{k}\right), i \neq j$, is assigned to the corresponding edge of $\mathscr{G}$. All edges with $\mathscr{M}_{i, j}>\varepsilon_{M}$ are deleted from $\mathscr{G}$. The Gaussian mixture components belonging to each maximal connected subgraph $\mathscr{C}_{\xi}$ of the remaining graph $\mathscr{G}^{\prime}$, are replaced by a Gaussian approximation $\tilde{f}_{x, k}^{e, \xi}\left(\underline{x}_{k}\right)$ with the same mean and covariance [17], [18].

To find out whether these replacements are acceptable the integral quadratic distance

$$
\mathscr{D}_{\text {quad }, \xi}=\int_{\mathbb{R}^{n}}\left(\sum_{j \in \mathscr{C}_{\xi}} \bar{f}_{x, k}^{e, j}\left(\underline{x}_{k}\right)-\tilde{f}_{x, k}^{e, \xi}\left(\underline{x}_{k}\right)\right)^{2} d \underline{x}_{k}
$$

is calculated for each subgraph $\mathscr{C}_{\xi}$. This distance measure is preferred over the Kullback-Leibler-like distances discussed in Section IV, because the integrand of (24) can be rewritten analytically as a Gaussian mixture, if the approximation error between a Gaussian and a Gaussian mixture density is calculated. Hence, this integral can always be solved analytically. In contrast to (24), all Kullback-Leibler-like distances contain the logarithm of a Gaussian. Therefore, in the merging step, the Kullback-Leibler-like distances always have to be integrated numerically.

\section{E. Calculation of the Prediction Step}

Up to now, only the filter step of the PDSME, consisting of the calculation of a measure for the linearization error, the splitting of the prior density, the calculation of the measurement update, and the merging step has been discussed. In this subsection, the prediction step of the PDSME, see the lower part of Fig. 1, is described. Analogously to the filter step, the state equation is approximated by a linearization

$$
\underline{a}_{k}\left(\underline{x}_{k}\right) \approx \underline{\bar{a}}_{k}\left(\underline{x}_{k}\right):=\underline{A}_{0}^{j}\left(\underline{\mu}_{x, k}^{e, j}\right)+\mathbf{A}_{1}^{j}\left(\underline{\mu}_{x, k}^{e, j}\right) \cdot \underline{x}_{k}
$$

at the means $\underline{\mu}_{x, k}^{e, j}$.

The linearization error corresponding to (9) is

$$
\begin{aligned}
\mathscr{D}_{2} & \left(\bar{f}_{x, k+1}^{p, j} \| f_{x, k+1}^{p, j}\right) \\
= & \int_{\mathbb{R}^{n}} \bar{f}_{x, k+1}^{p, j}\left(\underline{x}_{k+1}\right)\left(\ln \left(\frac{\bar{f}_{x, k+1}^{p, j}\left(\underline{x}_{k+1}\right)}{f_{x, k+1}^{p, j}\left(\underline{x}_{k+1}\right)}\right)\right)^{2} d \underline{x}_{k+1}
\end{aligned}
$$

with the approximated component

$$
\bar{f}_{x, k+1}^{p, j}\left(\underline{x}_{k+1}\right)=\int_{\mathbb{R}^{n}} \underbrace{f_{x, k}^{e, j}\left(\underline{x}_{k}\right) f_{w}\left(\underline{x}_{k+1}-\underline{a}_{k}\left(\underline{x}_{k}\right)\right)}_{\bar{f}_{x, k, k+1}^{p, j}\left(\underline{x}_{k}, \underline{x}_{k+1}\right)} d \underline{x}_{k}
$$

of the predicted PDF and the exact component

$$
f_{x, k+1}^{p, j}\left(\underline{x}_{k+1}\right)=\int_{\mathbb{R}^{n}} \underbrace{f_{x, k}^{e, j}\left(\underline{x}_{k}\right) f_{w}\left(\underline{x}_{k+1}-\underline{a}_{k}\left(\underline{x}_{k}\right)\right)}_{f_{x, k, k+1}^{p, j}\left(\underline{x}_{k}, \underline{x}_{k+1}\right)} d \underline{x}_{k} .
$$

Due to the convolution integral, the exact predicted density can only be evaluated numerically for nonlinear state equations $\underline{a}_{k}\left(\underline{x}_{k}\right)$. Therefore, the linearization error

$$
\begin{gathered}
\mathscr{D}_{2}\left(\bar{f}_{x, k, k+1}^{p, j} \| f_{x, k, k+1}^{p, j}\right)=\iint_{\mathbb{R}^{n}} \bar{f}_{\mathbb{R}^{n}} \bar{f}_{x, k, k+1}^{p, j}\left(\underline{x}_{k}, \underline{x}_{k+1}\right) . \\
\cdot\left(\ln \left(\frac{\bar{f}_{x, k, k+1}^{p, j}\left(\underline{x}_{k}, \underline{x}_{k+1}\right)}{f_{x, k, k+1}^{p, j}\left(\underline{x}_{k}, \underline{x}_{k+1}\right)}\right)\right)^{2} d \underline{x}_{k+1} d \underline{x}_{k}
\end{gathered}
$$

in the prediction step is defined for the approximation of the exact joint PDF component $f_{x, k, k+1}^{p, j}\left(\underline{x}_{k}, \underline{x}_{k+1}\right)$ in (28) by the Gaussian mixture component $\bar{f}_{x, k, k+1}^{p, j}\left(\underline{x}_{k}, \underline{x}_{k+1}\right)$ in (27). After this simplification, there is almost no difference to the filter step, except for that fact, that the joint density depends upon the state variables $\underline{X}=\left[\begin{array}{ll}\underline{x}_{k}^{T}, & \underline{x}_{k+1}^{T}\end{array}\right]^{T}$ with $\operatorname{dim}(\underline{X})=2 n$. Again, the component $j_{\max }$ of $f_{x, k}^{e}\left(\underline{x}_{k}\right)$ with the largest linearization error $\mathscr{D}_{2, \max }$ is replaced by $\left(L_{R}\right)^{n}$ Gaussian mixture components with smaller covariances. The parameters of each component of the predicted density are

$$
\begin{aligned}
\omega_{x, k+1}^{p, j} & =\omega_{x, k}^{e, j}, \\
\underline{\mu}_{x, k+1}^{p, j} & =\underline{A}_{0}^{j}+\mathbf{A}_{1}^{j} \underline{\mu}_{x, k}^{e, j}+\underline{\mu}_{w}, \text { and } \\
\mathbf{C}_{x x, k+1}^{p, j} & =\mathbf{A}_{1}^{j} \mathbf{C}_{x x, k}^{e, j}\left(\mathbf{A}_{1}^{j}\right)^{T}+\mathbf{C}_{w} .
\end{aligned}
$$

Afterwards, a merging step as in Subsection V-D is applied.

\section{Simulation Results}

\section{A. Scalar Filter Step}

The filter step of the PDSME by splitting the prior PDF to reduce the linearization error (9) is demonstrated for the scalar quadratic measurement equation

$$
\hat{y}_{k}=x_{k}^{2}+v_{k}
$$

with Gaussian measurement noise $v_{k}$. A Gaussian prior density with $\mu_{x, k}^{p}=1.0$ and $\sigma_{x, k}^{p}=1.0$ is assumed. In Fig. 3, the result of the filter step is shown for a fixed measured value $\hat{y}_{k}=0.75$ and Gaussian measurement noise with $\mu_{v}=$ 0.0 and $\sigma_{v}=0.5$. In the diagrams, the comparison of the 
approximated filter step to the exact posterior density is shown, after splitting the prior density into $L=400$ mixture components. For $L=1$, the result of the PDSME is equivalent to a standard EKF.
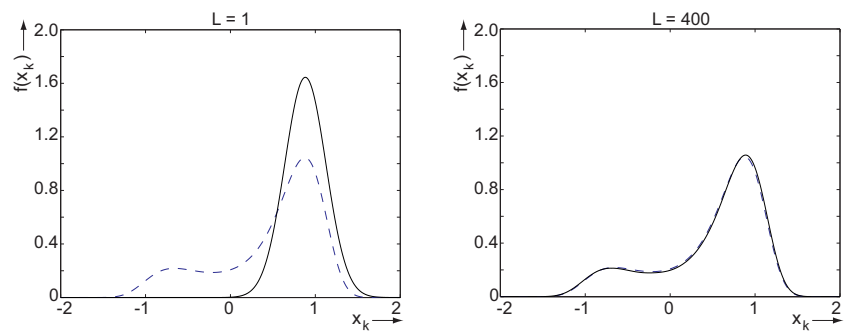

Fig. 3. Comparison of the exact posterior density (dashed lines) to a Gaussian mixture approximation (solid lines) in the filter step.

As mentioned in Section IV, for polynomial measurement equations (33), the linearization error

$$
\begin{gathered}
\mathscr{D}_{2}\left(\bar{f}_{x, k}^{e, j} \| f_{x, k}^{e, j}\right)=\int_{-\infty}^{\infty} \underbrace{f_{x, k}^{p, j}\left(\underline{x}_{k}\right) f_{v}\left(\hat{y}_{k}-\bar{h}_{k}\left(x_{k}\right)\right)}_{\bar{f}_{x, k}^{e, j}} \cdot \\
\cdot\left(-\frac{1}{2}\left(\frac{\hat{y}_{k}-\bar{h}_{k}\left(x_{k}\right)}{\sigma_{v}}\right)^{2}+\frac{1}{2}\left(\frac{\hat{y}_{k}-x_{k}^{2}}{\sigma_{v}}\right)^{2}\right)^{2} d x_{k}
\end{gathered}
$$

is expressed in terms of moments of the Gaussian PDF $\bar{f}_{x, k}^{e, j}$.

\section{B. Scalar Prediction Step}

In the prediction step, the nonlinear transformation

$$
x_{k+1}=x_{k}^{2}+w_{k}
$$

of a random variable $x_{k}$ with additive Gaussian system noise $w_{k}$ is considered. In this example, a Gaussian PDF $f_{x, k}^{e}\left(x_{k}\right)$ with $\mu_{x}^{e}=1.4$ and $\sigma_{x}^{e}=0.8$ has been assumed. The noise $w_{k}$ is described by $\mu_{w}=0.0$ and $\sigma_{w}=0.1$. In Fig. 4 , the result of the prediction step of the PDSME shows that already for $L=52$ the numerically calculated exact predicted PDF and its Gaussian mixture approximation are almost identical.
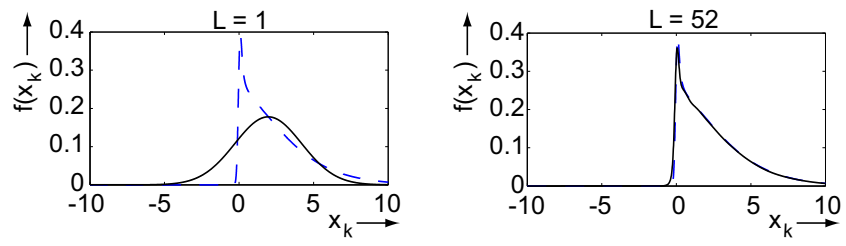

Fig. 4. Comparison of the exact predicted density (dashed lines) to approximations with different numbers of mixture components (solid lines).

\section{CONCLUSIONS}

In this paper, an efficient Gaussian mixture filtering algorithm has been proposed. This algorithm provides a solution for both the prediction and filter step. In contrast to other Gaussian mixture filtering algorithms, in which nonlinear measurement and state equations are replaced by linearizations at the means of the prior density components, the
PDSME is characterized by systematically splitting the prior density function using a pre-calculated optimized library of replacement densities. The components to be split into terms with smaller covariances, are selected by a Kullback-Leiblerlike measure for the linearization error. Re-approximation of the prior density by a Gaussian mixture with an increased number of components with smaller covariances reduces the linearization error and therefore improves the approximation quality, which can be specified by the user. By limiting the maximum number of mixture components, the maximum computational effort can also be specified. If the number of mixture components used by this algorithm is not limited, the result of the PDSME converges to the exact Bayesian state estimation. For finite numbers of mixture components, the quality of the PDSME is always superior to the Extended Kalman Filter, which only uses a single Gaussian component for the approximation of the posterior density.

\section{REFERENCES}

[1] A. Doucet, S. Godsill, and C. Andrieu, "On Sequential Monte Carlo Sampling Methods for Bayesian Filtering," Statistics and Computing, vol. 10, pp. 197-208, 2000.

[2] H. Tanizaki, "Nonlinear and Non-Gaussian State Space Modeling Using Sampling Techniques," Annals of the Institute of Statistical Mathematics, vol. 53, no. 1, pp. 63-81, 2001.

[3] M. S. Arulampalam, S. Maskell, N. Gordon, and T. Clapp, "A Tutorial on Particle Filters for Online Nonlinear/Non-Gaussian Bayesian Tracking," IEEE Transactions On Signal Processing, vol. 50, no. 2, pp. 174-188, February 2002.

[4] E. Bølviken, P. J. Acklam, N. Christophersen, and J.-M. Størdal, "Monte Carlo Filters for Non-linear State Estimation," Automatica, vol. 37, no. 2, pp. 177-183, 2001.

[5] A. Rauh and U. D. Hanebeck, "Nonlinear Moment-Based Prediction Step for Exponential Densities," in Proc. of the 44th IEEE Conference on Decision and Control and European Control Conference ECC 2005, Seville, Spain, 2005, pp. 1923-1928.

[6] A. Papoulis, Probability, Random Variables, and Stochastic Processes. Tokyo: McGraw-Hill, 1965.

[7] F. C. Schweppe, Uncertain Dynamic Systems. New York: PrenticeHall, 1973.

[8] K. Ito and K. Xiong, "Gaussian Filters for Nonlinear Filtering Problems," IEEE Transactions on Automatic Control, vol. 45, no. 5, pp. 910-927, 2000.

[9] R. E. Kalman, "A New Approach to Linear Filtering and Prediction Problems," Trans. ASME, J. Basic Eng., vol. Series 82D, pp. 35-45, 1960.

[10] D. Alspace and H. Sorenson, "Nonlinear Bayesian Estimation Using Gaussian Sum Approximations," IEEE Transactions on Automatic Control, vol. 17, pp. 439-448, 1972.

[11] N. Ueda, R. Nakano, Z. Ghahramani, and G. E. Hinton, "SMEM Algorithm for Mixture Models," Advances in Neural Information Processing Systems, vol. NIPS11, pp. 599-605, 1999.

[12] — "SMEM Algorithm for Mixture Models," Neural Computation, vol. 12, no. 9, pp. 2109-2128, 2000.

[13] S. Kullback and R. A. Leibler, "On Information and Sufficiency," Annals of Mathematical Statistics, vol. 22, pp. 79-86, 1951.

[14] H. Jeffreys, "An Invariant Form of the Prior Probability in Estimation Problems," Proc. Roy. Soc. A, vol. 186, pp. 453-461, 1946.

[15] D. H. Johnson and S. Sinanović, "Symmetrizing the KullbackLeibler Distance," Rice University, Tech. Rep., 2001, http: //cmc.rice.edu/docs/docs/ Joh2001Mar1Symmetrizi.pdf.

[16] P. C. Mahalanobis, "On the Generalized Distance in Statistics," Proc. Nat. Inst. of Sciences of India, vol. 2, no. 1, 1936.

[17] M. West, "Mixture Models, Monte Carlo, Bayesian Updating and Dynamic Models," Computing Science and Statistics, vol. 24, pp. 325333, 1993.

[18] M. West and P. J. Harrison, Bayesian Forecasting and Dynamic Models. New York: Springer-Verlag, 1997. 